\title{
Im Gleichgewicht
}

\author{
Roland Kopp-Wichmann
}

Work-Life-Balance wird mehr propagiert als praktiziert. Die Folgen sind oft verheerend, für den Einzelnen, die Familie, das Unternehmen, die Gesellschaft.

Niemand ist plötzlich ausgebrannt. Der Verlust an Kraft, Vitalität und Lebensfreude verläuft meist unauffällig und schleichend. Schlafstörungen, Konzentrationsschwierigkeiten und Stimmungsschwankungen können weitere Signale sein, die ernst zu nehmen sind.

Gerade Menschen, die bereit sind, für Ihre anspruchsvollen Ziele einen hohen Preis zu zahlen, können oft sich selbst und anderen schlecht eine Grenze setzen. Ihr Ehrgeiz treibt Sie zu Höchstleistungen, denn das Beste ist gerade noch gut genug für Sie. Der Einsatz, mit dem sie hartnäckig Ihre Ziele verfolgen, ist bemerkenswert. Ihre Erwartungen an sich und andere sind sehr hoch gesteckt. Doch selbst beim Erreichen eines Zieles tritt keine Entspannung oder Zufriedenheit auf. Zu schnell ist bereits das nächste Ziel im Blick.

Der Preis: Gefühle werden oft in den Hintergrund gedrängt und eigene Bedürfnisse werden kaum wahrgenommen. Das Leben verläuft wie in einem Hamsterrad. Kommen zu solchen inneren Dynamiken äußere Stressoren hinzu, ergibt sich ein gefährliches Zusammenspiel, das auch das stärkste Feuer zum Erlöschen bringen kann.

\section{Wie das Leben aus dem Gleichgewicht geraten kann}

Wahrscheinlich kennen Sie das: Für ein Projekt sind Überstunden nötig, zu Hause brauchen die Kinder Unterstützung bei den Schulaufgaben, im Garten ist einiges zu tun - und für das Wochenende haben sich Freunde angemeldet. Zeit, zu sich selbst zu finden oder mit dem Partner etwas zu unternehmen, fehlt schon seit Monaten. Was ist die Folge? Bei vielen Menschen kommt dann eine Gereiztheit auf, die sich einschleicht sowie das Gefühl, nicht mehr über die eigene Zeit bestimmen zu können, denn das Verhältnis zwischen Beruf- und Privatleben ist nicht mehr im Gleichgewicht.

Dies ist kein Einzelfall. Viele Menschen in fast allen Berufen und Branchen spüren in den letzten Jahren die gestiegene Arbeitsbelastung. Die Krankheitstage in Firmen nehmen ab, die stressbedingten Krankheitssymptome sowie die psychischen Störungen nehmen zu.

Was tun? Work-Life-Balance - heißt das neue Stichwort, das man immer öfter lesen kann. Arbeit und (Privat-) Leben wieder in Balance bringen? Doch wie geht das?

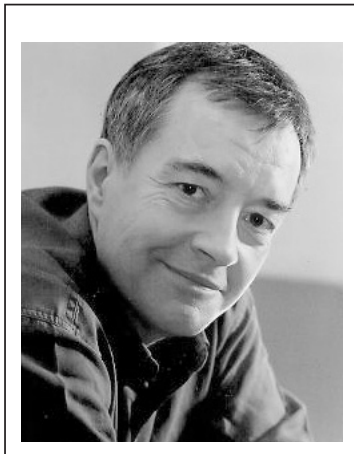

Roland Kopp-Wichmann ist gelernter Bankkaufmann und sammelte Berufserfahrungen in Werbung und Finanzdienstleistung sowie in der Leitung eines Instituts für Erwachsenenbildung. Nach dem Abitur auf dem zweiten Bildungsweg studierte er Psychologie und arbeitete ein Jahr in einem Kibbuz in Israel. Seit 1983 ist er als Trainer und Coach tätig. Ausbildungen hat er in Transaktionsanalyse, im systemischen Arbeiten, in tiefenpsychologischer und in Hypno- und Körpertherapie. In seinem intensiven Persönlichkeitsseminaren geht es um Themen wie emotionale Intelligenz, Gesprächsführung, Konflikt- und Selbstmanagement. Auf seiner Website bietet er neben Informationen zu seinen Seminaren auch einen eigenen »Podcast « mit Vorträgen zum Herunterladen an.

Internet http://www.seminare4you.de

\section{Wie man Fallen erkennt}

Wie kommt es zu einer einseitigen Betonung der Arbeit? Ein Grund ist, dass viel zu arbeiten für viele Menschen immer noch als wichtiger Wert und als Quelle von Anerkennung gilt. Aus diesem Grund finden auch bisweilen von Firmen angebotene Teilzeitmodelle bei Führungskräften und Mitarbeitern bis heute oft keine große Resonanz. Denn deutlich mehr zu arbeiten, wird oft mit besonderem Einsatz, Fleiß oder ausgeprägter Leistungsfähigkeit gleichgesetzt. Doch wer länger arbeitet, muss nicht effizient arbeiten. Ganz im Gegenteil: In meinen Zeitmanagementseminaren höre ich immer wieder Klagen über die Zeit,

- die in zu vielen Meetings sinnlos vertan wird,

- die beim Beantworten viel zu vieler E-Mails verloren geht oder

- bei Mehrfach- und Doppelarbeiten, die durch falsche oder ungenaue Anweisungen und schlechte Koordination verursacht werden.

Die Arbeitsdichte der unterschiedlichen Aufgaben und die Anforderungen im Beruf haben in den letzen Jahren in fast allen Berufen spürbar zugenommen. Eine Folge davon ist, dass die Zahl der Burn-out-Erkrankungen gestie- 
gen ist. Dabei trifft es nicht nur ältere Arbeitnehmer. Immer häufiger brennen auch Jüngere zwischen dreißig und vierzig Jahren aus. Vor einigen Jahren betraf diese Problematik vor allem Menschen ab fünfzig.

Burn-out-Gefährdete fixieren sich stark auf ihre Arbeit, wobei oft gleichzeitig ein Ausgleich durch Familie oder Hobbies fehlt. Gerade zu Beginn der Karriere nehmen Jüngere eine höhere Belastung in Kauf. Von den Unternehmen wird dies auch gern gesehen oder gar erwartet. Doch eine fehlende Work-Life-Balance führt am Ende statt zu mehr Leistung genau in die entgegengesetzte Richtung. Kurzfristig senkt verstärkter Einsatz oder der Versuch, über die eigene Leistungsgrenze zu gehen die Kosten, mittel- und langfristig können überbelastete Mitarbeiter für ein Unternehmen auch teuer werden. Ab einem bestimmten Punkt leistet der überforderte Mitarbeiter immer weniger, bis er ganz ausfällt - und das meist für längere Zeit. Laut Fraunhofer-Institut belaufen sich die Verluste durch Stress am Arbeitsplatz hierzulande auf jährlich mehr als drei Milliarden Euro.

Empirisch belegte Zahlen zum Phänomen »Arbeitssucht « fehlen zwar noch, doch vorsichtige Schätzungen gehen von rund 200.000 Arbeitssüchtigen in Deutschland aus. Als gefährdet gelten immerhin 13 Prozent aller Arbeitnehmer, Tendenz steigend. Im Prinzip ist jede intensive Arbeit, die auch emotionale Anforderungen stellt, eine potenzielle Gefahr auszubrennen. Burn-out kann die Folge von großen Engagement, Ehrgeiz und Leistungsdruck sein. Jeder, der dauerhaft unter Stress steht und sich einseitig auf die Arbeit fixiert, ist gefährdet. Diese Fokussierung ist oft ein Ausdruck für persönliche und soziale Unzufriedenheit. Irgendwann mündet der Zustand dann in körperliche und seelische Erschöpfung.

Was deutet darauf hin, dass Sie burn-out-gefährdet sein können? Diese Faktoren können aus einer Situation eine Burn-out-Gefahr machen:

- starkes emotionales Engagement

- Einzelkämpfertum, wenig Teamwork, starke Konkurrenz

- wenig Erfolgserlebnisse, wenig Wertschätzung

- permanent hoher Leistungsdruck, hohe Arbeitsbelastung, zu hohe innere oder äußere Anforderungen

- Fehlen von Fairness, Respekt, Gerechtigkeit, Erleben von Ungerechtigkeit

- wenig Veränderungs- oder Kontrollmöglichkeiten

Burn-out passiert nicht von einem Tag zum anderen, sondern es ist ein langsamer schleichender Prozess der einzelne, oft aber auch mehrere Mitarbeiter eines Betriebes befällt. Überhöhte Arbeitsbelastung, Stress sind neben geringer Teamfähigkeit und emotionalen Überengagement entscheidende Faktoren, die diese Gefahr fördern können. Werden diese Faktoren erkannt und durch entsprechende Gegenmaßnahmen verringert, kann mit Freude und Jobengagement auch viel an Arbeitseffizienz gewonnen werden.

Burn-out zeigt sich auf der körperlichen Ebene durch Symptome wie:

- Kopf- und Rückenschmerzen

- Blutdruckinstabilität

- Schlaf- und Sexualitätsstörungen

- vermehrte Anfälligkeit für Infekte
Auf der psychischen Seite können Hinweise für Burnout-Gefährdung sein:

- Unlust und Antriebslosigkeit

- depressive Symptome wie Schlafprobleme und Konzentrationsstörungen, Stimmungsschwankungen

- Angst vor Konflikten in der Arbeit

- eine »Alles-egal-Haltung « bis hin zum Zynismus

Krisenhafte Extremsituationen, körperliche Krankheit oder unvorhersehbare Ereignisse, können den Betroffenen vor einen Abgrund stellen, ihm aber auch den wesentlichen Impuls zur Veränderung geben. Wird eine Krise verdrängt und verleugnet, mit Alkohol und Tranquilizer »gedämpft «, mündet sie mitunter in einen chronischen, erschöpfenden und abstumpfenden Dauerzustand. Daher ist es besonders dann wichtig, wenn Sie glauben, es geht gar nichts mehr, alles hat sich zum Schlimmsten gewendet, besonders dann ist es wichtig, sich professionelle Hilfe zu holen.

\section{Wie man vier Aspekte unter einen Hut bekommt}

Viele Unternehmen zeigen zunehmend Interesse am Thema »Work-Life-Balance«, indem sie erkennen, dass es für beide Seiten Vorteile bringt, wenn die privaten Lebensverhältnisse der Mitarbeiter mit den beruflichen Erfordernissen abgeglichen werden. Firmen wie Bosch, Siemens und Ford praktizieren dabei aus durchaus eigennützigen Motiven betriebliche Instrumente wie Telearbeit, Teilzeitarbeit und Kinderbetreuung und andere Möglichkeiten.

Doch bei der Suche nach einer neuen Balance zwischen Job und Privatleben gibt es zwei Seiten. Hier das Unternehmen, das mit seinen eigenen Vorstellungen und Erfordernissen die Arbeitswelt und den Arbeitsplatz mitgestaltet. Auf der anderen Seite den Mitarbeiter, der neben der Organisation seiner Arbeit auch noch andere Prioritäten in seinem Leben hat. Dies miteinander in Einklang zu bringen, ist alles andere als leicht und die wichtigen Dinge werden oft genug von dringenden Aufgaben im Alltag verdrängt. Konkret heißt das: Nicht nur Unternehmen müssen umdenken und etliches verändern, auch die Menschen müssen ihre Einstellungen und Verhaltensweisen ändern.

Beim Finden einer neuen Work-Life-Balance geht es um vier Lebensbereiche:

- Arbeit und Leistung

- Körper und Gesundheit

- Familie und Soziales

- Sinn und Kultur

Ziele und Aufgaben in diesen vier Bereichen brauchen als gemeinsame Ressource den Faktor Zeit. Da jeder von uns nur 24 Stunden zur Verfügung hat, heißt das, dass wir tagtäglich die verschiedenen Erfordernisse und Wünsche gegeneinander abwägen müssen. Dabei hilft es uns Prioritäten zu setzen und zu lernen, Wichtiges von Dringlichem zu unterscheiden. Bei Letzterem hilft die Einsicht: Die wichtigen Dinge im Leben sind nicht dringend. Sie können es jedoch werden, wenn wir sie zu lange vernachlässigen. 
Der erste Schritt ist, sich über die eigene Lebenssituation bewusst zu werden. Hier sind Fragen hilfreich wie:

- Wie gestalte ich konkret mein Leben, um meine Leistungsfähigkeit langfristig zu erhalten?

- Welche Ziele habe ich in der Vergangenheit erreicht? Welche nicht?

- Wo stehe ich im Moment? Wie sind meine Situation, meine Gesundheit und meine innere Verfassung?

- Wie kann ich meinen persönlichen und beruflichen Erfolg sichern und eventuell ausbauen?

- Welche Ziele habe ich für die Zukunft - kurz-, mittel-, langfristig?

- Was stresst mich in meinem Leben? Wie kann ich besser damit umgehen?

- Was kann ich tun, um mein Leben mehr in meine Richtung zu lenken?

Doch diese Art der Selbstreflektion muss ein begleitender Prozess werden. Hilfreich ist dabei ein festes Ritual zu einem festen Zeitpunkt. Also zum Beispiel Tagebuchschreiben oder abends vor dem Einschlafen einige Zeit über den vergangenen Tag reflektieren.

\section{Wie man eine bessere Work-Life-Balance schafft}

1 Gehen Sie überlegt und ökonomisch mit Ihren 1. Kräften um. Auch wenn Sie es anfangs nicht glauben: Jeder Mensch hat nur begrenzte Energiereserven und muss seine Energiespeicher immer wieder auffüllen.

2 Achten Sie auf ihren Arbeitsablauf, erkunden Sie • Ihren persönlichen Tagesrhythmus und versuchen Sie, Ihren Stundenplan nach Ihrem persönlichen Biorhythmus einzuteilen. Ein Morgenmensch braucht einen anderen Ablauf als ein Abendmensch.

3.

Bauen Sie jede Stunde Kurzpausen ein - fünf Minuten genügen. Keine Zigarette! Wenn möglich bewe- gen Sie sich in dieser Zeit in der frischen Luft oder am offenen Fenster.

4. Gönnen Sie sich einmal im Verlauf des Arbeitstages 4. mindestens eine zwanzigminütige Pause, in der sie den Arbeitsplatz verlassen. Sie können Entspannungsübungen machen oder spazieren gehen. Wichtig ist, dass in dieser Zeit keinerlei neue berufliche Inhalte in Ihren Wahrnehmungskreis gebracht werden.

5 Vermeiden Sie Übermüdung. Bei Wechseldienst ist $\checkmark$. ausreichender Schlaf unbedingt nötig. Gewöhnen sie sich an, nach anstrengenden Ereignissen ein bis zwei Tage frei zu nehmen.

6 Alkohol, Beruhigungs- und andere Suchtmittel sind - keinesfalls geeignet, Stress abzubauen. Vermeiden Sie all jene Situationen, in denen deren Gebrauch zum Muss wird!

7 Sichern Sie sich einen Privatbereich, in dem berufli- che Belange Tabu sind. Auch die Familie, Freunde und Hobbys haben einen Platz in Ihrem Leben.

Q Sprechen Sie über Ihre Probleme mit den Kollegin8. nen und Kollegen. Es kann erleichtern, wenn man merkt, dass andere dieselben Sorgen haben.

Q Suchen Sie sich einen beruflichen Wirkbereich, in - dem Sie die Kontrolle behalten. Das kann etwas ganz Kleines, wie die Gestaltung des Arbeitsplatzes, sein.

10 Bestehen Sie auf adäquater Entlohnung, Wert1U. schätzung durch Vorgesetzte und einen menschlichen Umgang miteinander. Setzen Sie sich für eine gute Gesprächskultur an Ihrem Arbeitsplatz ein.

Seminare und Coaching können helfen, Zusammenhänge zu erkennen, die zu einem ungesunden Ungleichgewicht oder einem Burn-out-Syndrom geführt haben, um daraus dann notwendige Schritte abzuleiten. Dabei geht es auch darum, eine individuelle Work-Life-Balance zu finden, die bei jeder Person anders aussieht.

\section{Führen mit dem Eisenhower-Prinzip}

Eine nach dem General und späteren US-Präsidenten benannte Methode zur Entscheidungsfindung ist das Eisenhower-Prinzip. Mit ihm kann festgelegt werden, in welcher Reihenfolge Aufgaben am besten zu erledigen sind. Danach werden Prioritäten aufgrund der beiden Kriterien Dringlichkeit und Wichtigkeit vergeben. Daraus lassen sich dann Hinweise zum Umgang mit den jeweiligen Anforderungen ableiten.

\begin{tabular}{|l|ll} 
wichtig & $\begin{array}{l}\text { Aufgaben wenn möglich } \\
\text { delegieren oder später } \\
\text { selbst erledigen }\end{array}$ & $\begin{array}{l}\text { Aufgaben } \\
\text { sofort selbst } \\
\text { erledigen }\end{array}$ \\
\hline unwichtig & Aufgaben ablehnen & $\begin{array}{l}\text { Aufgaben wenn } \\
\text { möglich delegieren }\end{array}$ \\
& nicht dringend & dringend
\end{tabular}

